\title{
Terapia floral e sua representação social para professores de um centro universitário
}

\author{
Floral therapy and its representation social for teachers of a university center \\ La terapia floral y su representación social para profesores de un centro universitario
}

Recebido: 19/07/2021 | Revisado: 23/07/2021 | Aceito: 24/07/2021 | Publicado:31/07/2021

Luiz Carlos Moraes França
ORCID: https://orcid.org/0000.0002.6370-115X
Centro Universitário Anhanguera de Niterói, Brasil
E-mail: lcmoraesfranca@ hotmail.com
Antônio Marcos Tosoli Gomes
ORCID: https://orcid.org/0000-0003-4235-9647
Universidade do Estado do Rio de Janeiro, Brasil
E-mail: mtosoli@gmail.com
Rachel Verdan Dib
ORCID: https://orcid.org/0000-0001-9684-1979
E-mail: rachelvdib@gmail.com
Pablo Luiz Santos Couto
Instituto Nacional de Câncer José Alencar Gomes da Silva, Brasil
ORCID: https://orcid.org/0000-0002-2692-9243
Centro Universitário Guanambi, Brasil
E-mail: pabloluizsc@ hotmail.com
Virginia Paiva Figueiredo Nogueira
ORCID: https://orcid.org/0000-0001-7331-9715
Universidade do Estado do Rio de Janeiro, Brasil
E-mail: virginiafigueiredo@ yahoo.com.br
Leandra da Silva Paes
ORCID: https://orcid.org/0000-0002-4742-1860
Universidade do Estado do Rio de Janeiro, Brasil
E-mail: lepaes80@yahoo.com.br
Larissa Alves Moura de Moraes
ORCID: https://orcid.org/0000-0001-5190-9183
Centro Universitário Anhanguera de Niterói, Brasil
E-mail: Larissa_shou @ hotmail.com
Juliana Rodrigues da Silva Gomes
ORCID: https://orcid.org/0000-0002-5663-8981
Centro Universitário Anhanguera de Niterói, Brasil
E-mail: julianar177@gmail.com

\section{Resumo}

Objetivo: Analisar os conteúdos que integram a representação social da terapia floral em sua análise estrutural para professores de um centro universitário. Metodologia: Trata-se de um estudo qualitativo, descritivo e exploratório, pautado na Teoria das Representações Sociais em sua abordagem estrutural com 66 professores universitários de um Centro Universitário localizado no município de Niterói, Rio de Janeiro. Foi coletado um questionário sociodemográfico e a técnica de evocação livre ao termo indutor "Terapia Floral" via Google Forms. Critérios de inclusão: Participação voluntária por meio da assinatura do Termo de Consentimento Livre e Esclarecido (TCLE). Resultados e Discussão: O grupo compreende a terapia floral como algo proveniente da natureza, através do uso de essências, com potencial alternativo para tratamento. O termo natureza e essência, presentes no possível núcleo central é reforçado pelas demais periferias, como confirmado pela análise de similitude, que mostra natureza como sendo o elemento com ligações mais fortes da representação. Conclusão: Há carência de estudos sobre a temática, reforçando a importância de mais publicações, capacitação e treinamento de profissionais para a utilização dessa prática já autorizada legalmente para realização no Sistema Único de Saúde.

Palavras-chave: Terapia floral; Representações sociais; Professores universitários.

\begin{abstract}
Objective: To analyze the contents that integrate the social representation of flower essence therapy in its structural analysis for professors at a university center. Methodology: This is a qualitative, descriptive and exploratory study, based on the Theory of Social Representations in its structural approach with 66 university professors from a University Center located in the city of Niterói, Rio de Janeiro. A sociodemographic questionnaire and the technique of free evocation of the inducing term "Floral Therapy" via Google Forms were collected. Inclusion criteria: Voluntary participation by signing the Informed Consent Form (TCLE). Results and Discussion: The group
\end{abstract}


understands flower essence therapy as something from nature, through the use of essences, with alternative potential for treatment. The term nature and essence, present in the possible central nucleus, is reinforced by the other peripheries, as confirmed by the similarity analysis, which shows nature as the element with the strongest connections in the representation. Conclusion: There is a lack of studies on the subject, reinforcing the importance of more publications, training and training of professionals for the use of this practice already legally authorized to be carried out in the Unified Health System.

Keywords: Floral therapy; Social representations; University professors.

\begin{abstract}
Resumen
Objetivo: Analizar los contenidos que integran la representación social de la terapia de esencias florales en su análisis estructural para profesores de un centro universitario. Metodología: Se trata de un estudio cualitativo, descriptivo y exploratorio, basado en la Teoría de las Representaciones Sociales en su enfoque estructural, con 66 profesores universitarios de un Centro Universitario ubicado en la ciudad de Niterói, Rio de Janeiro. Se recogió un cuestionario sociodemográfico y la técnica de evocación libre del término inductor "Terapia Floral" a través de Google Forms. Criterios de inclusión: Participación voluntaria mediante la firma del Formulario de Consentimiento Informado (TCLE). Resultados y discusión: El grupo entiende la terapia de esencias florales como algo de la naturaleza, mediante el uso de esencias, con potencial alternativo de tratamiento. El término naturaleza y esencia, presente en el posible núcleo central, es reforzado por las otras periferias, como lo confirma el análisis de similitud, que muestra a la naturaleza como el elemento con mayores conexiones en la representación. Conclusión: Existe una falta de estudios sobre el tema, lo que refuerza la importancia de más publicaciones, formación y formación de profesionales para el uso de esta práctica ya legalmente autorizados para ser realizados en el Sistema Único de Salud.
\end{abstract}

Palabras clave: Terapia floral; Representaciones sociales; Profesores universitarios.

\title{
1. Introdução
}

A Terapia Floral é considerada uma Prática Integrativa Complementar em Saúde (PICS) e sua fundamentação apresenta grande cunho na prática psicológica e espiritual, atuando no equilíbrio do corpo humano e sendo um potencializador da saúde. Constituída por essências florais derivadas de plantas, é uma prática complementar não medicamentosa, de característica não invasiva, segura e com eficácia evidenciada na literatura capaz de agregar uma série de benefícios ao Sistema Único de Saúde (SUS) (Brasil, 2006; Nascimento, et al., 2017).

Os florais tiveram sua origem a partir do médico Edward Bach, nascido no ano de 1886, em Moseley, uma pequena vila próxima de Birmingham, na Inglaterra. Edward trabalhou em um departamento de bacteriologia e imunologia após a sua formação. Durante a atuação na profissão, percebeu que os pacientes reagiam de forma individual aos tratamentos, visto que uns atingiam a cura e outros não ao receberem a mesma terapêutica. Em 1917, Edward ficou extremamente doente, entendendo que a cura que o paciente obtém está intimamente ligada ao seu estado físico e emocional. Alguns anos depois, após muito estudo, desenvolveu um total de 38 essências que são utilizadas a nível mundial (Batistella, et al., 2021; Nascimento 2017).

No Brasil, essa terapia tem ganhado evidência desde a aprovação da Política Nacional de Práticas Integrativas Complementares (PNPIC), em 2006. A Organização Mundial da Saúde (OMS) reconhece a importância da prática da terapia floral, incentivando sua implementação nos Sistemas Nacionais de Saúde, corroborando para a integralidade da assistência (Brasil, 2006).

A terapia floral não se contrapõe a nenhum tratamento médico, sendo utilizada em seu potencial para os problemas que afetam os seres humanos, como estresse, ansiedade e insônia, por exemplo, sem interferir no diagnóstico ou indicação terapêutica habitual. Em seu objetivo, a terapia floral, ajuda nos casos em que a medicina tradicional muitas vezes não encontra solução, pois ela trata apenas o sintoma e não a causa que leva a motivação da doença ou do seu desequilíbrio pessoal (Vasconcelos, 1997).

Se reconhece, portanto, que existe uma demanda extensa por parte da sociedade em geral para que as necessidades relacionas a problemas do cotidiano sejam supridas de alguma maneira. Contudo, reside um desafio e missão no meio acadêmico, seja em decorrência da prática de ensino, pesquisa e extensão que é, por assim dizer, adicionada às demandas pessoais desses indivíduos (Júnior, et al., 2019; Pinto, et al., 2020). 
Entre as profissões consideradas estressantes, encontra-se a do professor universitário, que exige uma carga horária de trabalho para além da sala de aula para realização de atividades, número reduzido de horas dormidas, falta de atividades extraclasse como recreação, além da estrutura física e relações humanas do local de trabalho, contribuem para que esse profissional apresente uma carga de estresse importante, tendo como consequência risco de adoecimento e redução da qualidade de vida (Júnior, et al., 2019; Pinto, et al., 2020).

No campo do ensino, por exemplo, devemos não apenas formar profissionais aptos ao diálogo técnico, devemos estar habilitados aos mais variados assuntos dentre nossas atividades desempenhadas. Dessa forma, compreender o universo consensual desses professores através das representações sociais sobre a terapia floral, nos parece proveitoso.

Acerca da Teoria das Representações Sociais (TRS), segundo Moscovici (1978), a representação social, como modalidade de conhecimento particular, tem por função orientar comportamentos e facilitar a comunicação entre os indivíduos, levando em conta a experiência subjetiva e a inserção social dos sujeitos. Esta se mostra influenciada a partir da percepção de mundo, cultura e a própria relação do senso comum para com o objeto (Alaya, 2019).

Diante do exposto, desenvolveu-se o presente estudo que tem por objetivo analisar os conteúdos que integram a representação social da terapia floral em sua análise estrutural para professores de um centro universitário no município do Rio de Janeiro, Brasil.

\section{Metodologia}

O presente estudo apresenta uma análise qualitativa, descritiva e exploratória, à luz da TRS em sua abordagem estrutural conforme descreve o autor supracitado Moscovici. Esse tipo de análise gera um quadro de quatro casas a partir da técnica de evocação livre, realizada através de um termo indutor, sendo "Terapia Floral" o termo utilizado nesta pesquisa, realizado em um Centro Universitário situado no município de Niterói, Brasil. Participaram da pesquisa um grupo de 66 professores universitários que trabalham na instituição escolhida para a realização do estudo. Os critérios de inclusão utilizados foram: aceite de colaborar com o estudo, assinando o Termo de Consentimento Livre e Esclarecido (TCLE) de forma voluntária.

A coleta de dados se deu de maneira remota, em ambiente virtual, explicando ao participante acerca da temática a ser estudada, marcando com este um horário de sua preferência. Foram enviados questionários contendo a caracterização sociodemográfica, conteúdo sobre práticas religiosas e o termo indutor "Terapia Floral" por meio do questionário de evocação livre para preenchimento via Google Forms.

A partir do termo evocado, o gera-se o quadro com quatro quadrantes, a saber: provável núcleo central localizado no quadrante superior esquerdo, primeira periferia situada na parte superior direita, zona de contraste encontrada no quadrante inferior direito e segunda periferia em quadrante inferior direito. Esta contém as palavras organizadas de acordo com sua frequência e Ordem Média de Evocação (OME). Essa abordagem permite a recognição do possível núcleo central contido na representação do grupo, como também a apresentação dos demais elementos contidos nas periferias (Sá, 2002; Wolter; Wachelke, 2016).

Após a coleta das evocações livres mediante ao termo indutor, registram-se os dados em uma planilha no Microsoft Excel. Estes dados tabulados serão posteriormente analisados pelo software Interface de $\mathrm{R}$ pour les Analyses Multidimensionnelles de Textes ET de Questionnaires (IRAMUTEQ), que se trata de um software de acesso gratuito, que atua por meio da análise de texto, ou de léxicos contidos em entrevistas e/ou questionários (Camargo \& Justo, 2013).

No que se refere a análise de similitude ou análise de semelhanças, essa estuda as relações entre os léxicos por meio da co-ocorrência existente entre as palavras evocadas pelos participantes do estudo, gerando então, uma nuvem de palavras por meio de um gráfico (Camargo \& Justo, 2017). A pesquisa em questão foi aprovada pelo Comitê de Ética em Pesquisa (CEP), 
sob o parecer de número 4.728 .067 e o Certificado de Apresentação para Apreciação Ética (CAAE) número 43524421.1.0000.5493.

\section{Resultados e Discussão}

O estudo compreende um total de 66 participantes, majoritariamente do sexo feminino 40 (60,6\%), contendo 31 pessoas, ou seja, (47\%) com intervalos de idade de 25 a 39 anos 40 a 59 anos. A maior parte do grupo (33 pessoas) informaram possuir estado civil casado (50\%), enquanto 33,3\% ou 22 participantes se declararam solteiros. Um número de 63 professores afirma acreditar em Deus (95,5\%), 33 do total de participantes se declararam de religião católica (50\%), 15 (22,7\%) da religião evangélica, $8(12,1 \%)$ kardecistas enquanto $5(7,6 \%)$ se afirmaram não seguir nenhuma religião.

A fim de se obter a representação social dos participantes, se utilizou a análise prototípica através do software IRAMUTEQ, sendo detectadas um total de 198 léxicos evocados, sendo 82 destas distintas. Optou-se por empregar uma frequência média igual a 8,07, frequência mínima de valor 4 e uma O.M.E de 1,91. A tabela a seguir (Tabela 1) ilustra os resultados obtidos após a estruturação dos dados:

Tabela 1: Quadro de quatro casas mediante a evocação livre do termo indutor "Terapia Floral" - Rio de Janeiro (RJ), Brasil.

\begin{tabular}{|l|l|l|l|l|l|l|}
\hline \multicolumn{2}{|l|}{ O.M.E. $<1,88$} & O.M.E. > = 1,88 & O.M.E. \\
\hline $\begin{array}{l}\text { Freq. } \\
\text { méd }\end{array}$ & Termo evocado & Freq. & O.M.E. & Termo evocado & Freq. & \\
\hline \multirow{3}{*}{$\geq 8,14$} & Natureza & 17 & 1,6 & Tratamento & 11 & 2 \\
& Essência & 14 & 1,6 & Alternativa & 11 & 2 \\
\hline \multirow{5}{*}{$\leq 8,14$} & Remédio & 8 & 1,5 & Equilíbrio & 8 & 2,2 \\
& Não Conheço & 7 & 1,5 & Calma & 7 & 2,4 \\
& Cuidado & 4 & 1,8 & Dúvida & 7 & 2,1 \\
& Placebo & 4 & 1 & Saúde & 7 & 2,3 \\
& & & & Tranquilidade & 5 & 2,2 \\
& & & & Melhora & 4 & 2 \\
\hline
\end{tabular}

Fonte: Dados da pesquisa (2021).

A análise do quadro exposto revela a representação social expressa pelos professores universitários que fizeram parte do estudo acerca do termo indutor "Terapia Floral". No quadrante superior esquerdo, onde se apresenta o possível núcleo central, se encontram os termos natureza e essência, sendo realçado pelos elementos contidos na primeira periferia tratamento e alternativa, localizados no quadrante superior direito, trazendo também significação ao núcleo central.

Para Bach (2006), a doença é a forma que a natureza encontra para chamar a atenção para o indivíduo, para acontecimentos que possam estar errado, como um sentido de harmonizar a personalidade com a alma, sendo assim um método efetivo. Neste sentido, ao referir à terapia floral, como natureza, considera-se que ela se torna um facilitador para esse encontro em busca dessa harmonia, em consonância com outros cognemas citados, como essência e sendo assim uma alternativa a tratamentos, inclusive medicamentosos.

Dentre algumas doenças crônicas, como por exemplo, diabetes, hipertensão arterial sistêmica, doenças cardiovasculares e outras condições ligadas a enfermidades que provocam sofrimento, percebe-se que as PICS, incluindo a terapia floral, são uma importante ferramenta para a prevenção, controle e tratamento dessas patologias, corroborando os achados no presente estudo (Mendes, 2012).

No que se refere à primeira periferia, no quadrante superior direito, se encontram os cognemas tratamento e alternativa, que reforçam o emprego da terapia floral como uma opção de tratamento de diversas demandas psicológicas e 
espirituais, que envolvem por exemplo a ansiedade e a depressão, tendo a terapia floral se apresentado como uma eficiente terapêutica a base de essência.

Ao ser identificada como uma abordagem terapêutica, a terapia floral utiliza essências florais na prevenção, tratamento e manutenção do equilíbrio emocional e psicológico de qualquer pessoa, de forma a favorecer a restauração da paz, harmonia e equilíbrio do ser humano. Segundo Sheffer (1997) a partir da relação entre as emoções humanas e a energia das flores, o uso das essências poderiam ser uma prática simples, por meio dos florais.

As palavras contidas na segunda periferia, encontrada no quadrante inferior direito evidencia uma circunstância iminente e atual identificada como consequência das demandas apresentadas pelo grupo através dos termos equilíbrio, calma, dúvida, saúde, tranquilidade, melhora.

Por fim, os termos presentes no quadrante inferior esquerdo, ou seja, na zona de contraste, exibem a ideia de a terapia floral estar associada ao tratamento de um estado de saúde, seja ele físico, emocional ou espiritual representado pelas palavras remédio e cuidado. Em contrapartida, vê-se uma contradição acerca da terapia de fato atuar com um papel medicinal por meio da palavra placebo e não conheço. Destarte, a integralidade seria um atributo usado também no contexto da atenção à saúde, sendo um qualificador de uma ação interpretativa e terapêutica, preventiva ou "curativa", integrando as múltiplas dimensões da vida dos sujeitos (Tesser; Luz, 2008).

Como descrito anteriormente, a análise de semelhanças é capaz de identificar as conexões presentes entre os léxicos que correspondem a representação social do grupo, analisando também suas conexões em cada âmbito de forma individualizada, gerando interpretações sobre os termos evocados pelos participantes acerca da terapia floral.

Conforme análise de similitude (Figura 1) abaixo, e os elementos contidos no quadro de quatro casas (Tabela 1), mostrando suas relações e organizações que contribuem com o provável núcleo central e suas periferias presentes na representação, discutiremos a seguir. 
Figura 1: Análise de similitude dos conteúdos evocados com o termo indutor "terapia floral" - Rio de Janeiro (RJ).

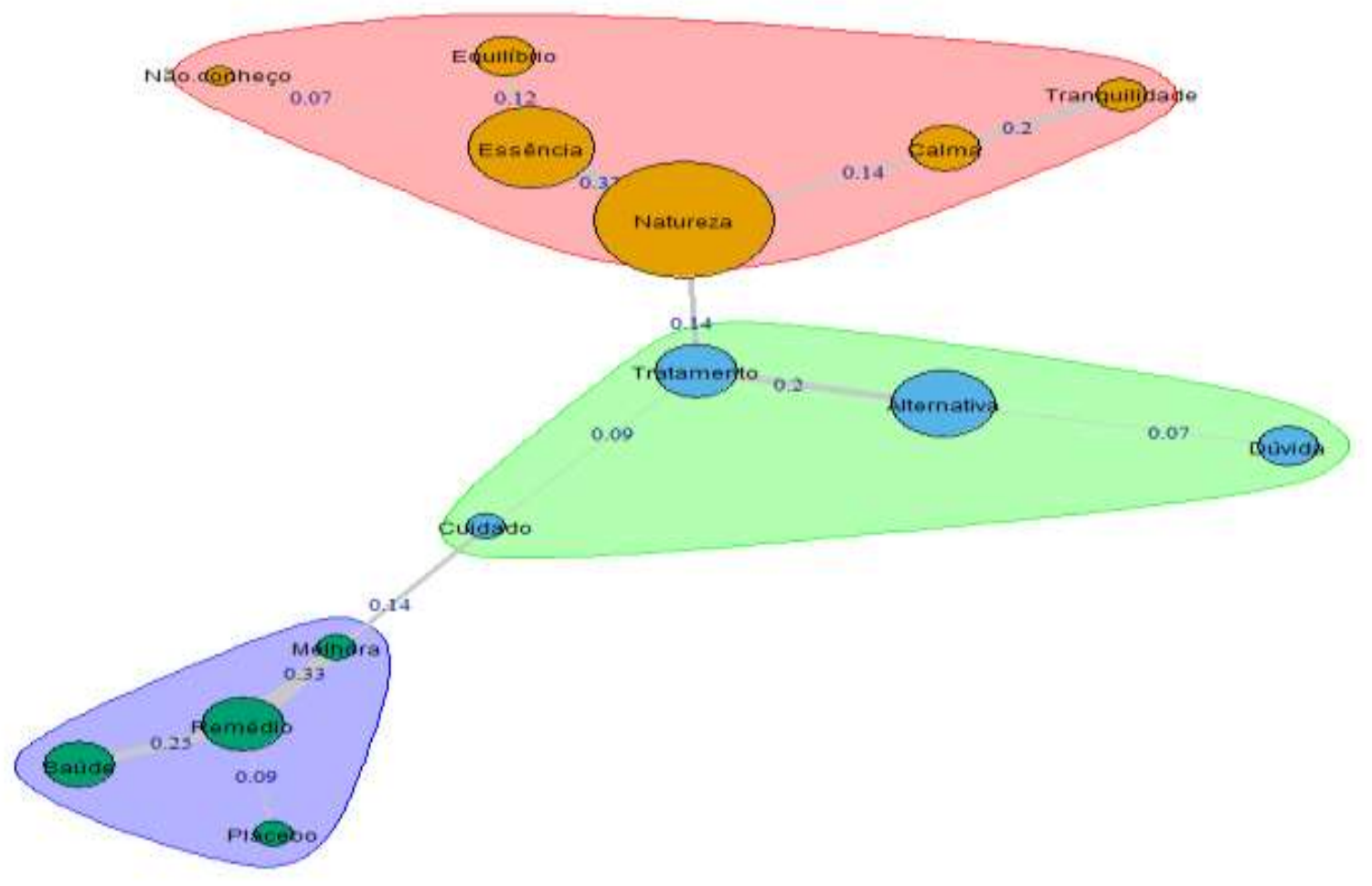

Fonte: Dados da pesquisa (2021).

Se pode perceber que o cognema natureza possui em sua organização o maior número de conexões, tendo uma forte ligação com a palavra essência, também apresentando conectividade com os elementos tratamento, calma. Sua expressiva conectividade mostra que há um importante indício de centralidade na representação do grupo ao reforçar o elemento pertencente ao possível núcleo central (natureza e essência). Ainda nesse eixo, nota-se que o termo essência está associado a uma forma de indicação da terapia como o equilíbrio, e a outro elemento identificado por não conheço, o que sugere o pouco conhecimento, até mesmo não divulgação da terapia floral no meio acadêmico.

A partir do cognema natureza, essa terapia floral conforme a análise se apresenta como uma alternativa, entretanto se observa a expressão dúvida, o que pode estar relacionado a incerteza sobre a terapêutica.

Ainda em análise, outro eixo que se destaca é o termo melhora, ligado ao cognema cuidado, podendo ser entendido como um cuidado em saúde em busca de melhora, sendo mais bem explicado com outros termos remédio, saúde. Contudo, outro termo em controvérsia aparece nesse eixo, sendo apresentado como placebo.

Muitas terapias alternativas trabalham com um enfoque subjetivo, onde a ação se dá a partir da visão do ser humano ao seu redor, em seu meio social, indo além da anatomia física, onde age com uma força energética complexa e sutil que regula sua mente, suas emoções, suas faculdades psíquicas e espirituais, o que pode ser percebido como algo duvidoso, ou até mesmo ineficaz por alguns (Patricio, 1992).

De acordo com Araújo et al., (2015) as PICS utilizam abordagem integral da pessoa, sendo baseada nas necessidades individuais, de forma a considerar os aspectos físico, mental, social, espiritual e emocional. Neste esteio, essas práticas colaboram para o aumento do bem-estar, promovendo o relacionamento com a sociedade e estimulando o autocuidado das pessoas em tratamento. 
No estudo de Salles e Silva (2012), com pessoas em uso de terapia floral, $80 \%$ do grupo referiu que, mesmo diante de eventos estressantes, conseguiram manter-se calmos, com clareza de ideias e concentração; $60 \%$ citaram menor irritação e impaciência com situações que normalmente os incomodavam e $40 \%$ melhoraram o padrão de sono. De acordo com o evidenciado acima, sugere que de acordo com a prática docente, o que muitas vezes se depara com eventos estressantes, a terapia floral surge como um facilitador nesse processo da prática profissional, envolvendo as experiências físicas, mentais, sensoriais e emocionais (Goswami, 2018).

Botelho e Sorato (2012) utilizaram em seus estudos uma mistura de três sistemas florais (Bach, australiano e californiano) no estresse do próprio docente universitário, observando uma diminuição média de $42,86 \%$ do estresse dos participantes do estudo, o que de acordo com o perfil dos professores, poderá ser uma terapia facilitadora.

Um estudo realizado com o uso de florais de Bach, em que se avaliou o alívio de estresse de profissionais de enfermagem que exerciam suas atividades em um cenário de emergência, Salomé et al (2009), observou o Reiki, a música e os florais de Bach como métodos de alívio de alterações físicas e frustrações dos profissionais. Os principais efeitos constatados foram a diminuição do estresse e do cansaço.

Em outra pesquisa, foi avaliada a motivação de pacientes no uso dos florais de Bach em tratamento de saúde, percebeu-se que as motivações em grande parte dos entrevistados, foram problemas do tipo emocional, como, insegurança, tristeza e ansiedade. A maioria dos participantes mostrou-se satisfeita com tratamento, referindo as expectativas atendidas, e apontando o desejo de continuar o uso dos florais devido a satisfação com o tratamento (Valente, 2011).

As práticas integrativas e complementares têm em comum o olhar holístico e o respeito pela individualidade, o que sugere a terapia floral, como um facilitador desse processo, podendo ser um aliado a prática docente, diante de suas atividades de ensino, seja presencial e até mesmo remoto, atuando em diversas situações cotidianas.

\section{Considerações Finais}

A terapia floral vem ganhando um espaço significativo em sua utilização no mundo, principalmente como uma opção de tratamento alternativo. A comprovação da sua efetividade e importância são destacadas pela PNPIC, sendo uma terapia que vem sendo implementada aos poucos no SUS. Há a necessidade de mais profissionais que se apoderem sobre os estudos já existentes e suas práticas, contribuindo para haja uma ampla utilização no SUS através de profissionais capacitados e pautados na ciência.

O conhecimento da terapia floral se mostrou bastante benéfica, porém ainda se identifica um certo desconhecimento sobre sua existência, objetivos e utilização, o que reforça a importância da difusão de informações baseadas em estudos científicos, comprovando sua eficácia e seu impacto na qualidade de vida daqueles que fazem uso da terapia.

Centros universitários podem ponderar sobre a incorporação do tema em cursos da área da saúde visando o contato desses com a temática, gerando interesse e possibilidade de novos estudos. Da mesma maneira, devem considerar a possibilidade de proporcionar um espaço para a prática da terapia floral para os docentes, buscando melhores respostas referentes ao nível de estresse dos professores universitários, possivelmente gerando um feedback positivo quanto a maior produtividade no trabalho, menor adoecimento e maior satisfação com o trabalho.

As limitações encontradas na execução do estudo perpassaram sobre o panorama mundial acerca da Covid-19, impossibilitando a coleta de dados de forma presencial. Além disso, pôde-se evidenciar por meio dos estudos publicados sobre o saldo positivo que a terapia floral oferece para aquele que a utiliza, reconhecendo grandes ganhos ao ser difundida no meio universitário. 
Urge a necessidade da realização de mais estudos acerca do tema, para que cada vez mais a prática seja conhecida e utilizada, além de novas pesquisas atestando o quão benéfico se mostra o uso da terapia floral a longo prazo, corroborando com os poucos dados científicos já existentes acerca dos resultados decorrentes da utilização dessa prática.

\section{Referências}

Alaya, D. B. Abordagens filosóficas e a teoria das representações sociais. Teoria das representações sociais, 50, 261-81, 2019.

Araújo, C. L. F. et al. (2015). Conhecimento e expectativas das pessoas que vivem com HIV/ Aids sobre as terapias complementares em saúde. In: VHI/sida: Experiências da doença e cuidados em saúde, Rio de Janeiro: Edições Humus.

Batistela, C. E. et al. (2021). Efetividade da terapia floral para redução de sintomas de ansiedade em universitários: ensaio clínico randomizado. Research, Society and Development. 10(1), e44710111926.

Botelho, S. H. \& Soratto, M. T. (2012). A terapia floral no controle do estresse do professor enfermeiro. Saúde Rev. 12(31), 31-42. https://www.metodista.br/revistas/revistas-unimep/index.php/sr/article/ download/1055/934.

Brasil. Ministério da Saúde. (2006). Secretaria de Atenção à Saúde. Departamento de Atenção Básica. Política Nacional de Práticas Integrativas e Complementares no Sistema Único de Saúde (PNPIC/SUS). Ministério da Saúde.

Bach, E. (2006). Os remédios florais do Dr. Bach incluindo cura-te a ti mesmo. Pensamento.

Camargo, B. V. \& Justo, A. M. (2013). Iramuteq: um software gratuito para análise de dados textuais. Temas em Psicologia, 21(2), 513-518. 10.9788/TP2013.2-16.

Camargo, B. V. \& Justo, A. M. (2017). Tutorial para uso do software iramuteq (interface de $r$ pour les analyses multidimensionnelles de textes et de questionnaires). Laboratório de Psicologia Social da Comunicação e Cognição - UFSC.

Cruvinel, R. H. J. et al. (2019). Níveis de estresse e variabilidade da frequência cardíaca em professores universitários. Revista Interdisciplinar de Estudos em Saúde, 9, 73-82.

Ferreira, N. V. et al. (2017). Utilização De Florais De Bach Na Psicoterapia Holística. Saúde.com, 13, 770-778.

Goswani, A. (2018). Consciência quântica: uma nova visão sobre o amor, a morte e o sentido da vida. Aleph.

Henrique, R. et al. (2019). Níveis de estresse e variabilidade da frequência cardíaca em professores universitários. Revista Interdisciplinar de Estudos em Saúde, 73-82.

Mendes E. V. (2012). O cuidado das condições crônicas na atenção primária à saúde: o imperativo da consolidação da estratégia da saúde da família. Brasília: Organização Pan-Americana da Saúde.

Moscovici, S. (1978). Social cognition: perspectives on everyday understanding. London (UK): Academic Press.

Patricio M. Z. (1992). Repensando paradigmas de saúde. Texto Contexto Enferm; 142-51.

Pinto, R. H. et al. (2020). Efeito da terapia floral no estresse docente: ensaio clínico randomizado. Reme Revista Mineira de Enfermagem, v. 24 , p. 1-8.

Sá, C. P. (2002). Núcleo central das representações sociais. (2a ed.), Vozes.

Salomé, G. M.. et al. (2020). Sentimentos vivenciados pelos profissionais de enfermagem que atuam em unidade de emergência. Rev. bras. enferm., 62, 856862.

Scheffer, M. (1997). Terapia floral do Dr. Bach: teoria e prática. (7a ed.), Pensamento.

Tesser, C. D., \& Luz, M. T. (2008). Racionalidades médicas e integralidade. Ciên. Saude Colet., 13, 195-206.

Vasconcelos, E. M. R. (1997). Uso dos Florais de Bach por indivíduos na terceira idade em fase depressiva: assistência de enfermagem. Universidade Federal da Paraíba.

Valente, M. J. C. (2011). A motivação dos pacientes no uso dos florais de Bach como prática complementar em tratamentos de saúde.

Wolter, R. P., Wachelke, J., \& Naiff, D. (2016). A abordagem estrutural das representações sociais e o modelo dos esquemas cognitivos de base: perspectivas teóricas e utilização empírica. Temas em Psicologia. 